\title{
Anticancer immune reactivity and long-term survival after treatment of metastatic ovarian cancer with dendritic cells
}

\author{
SAMUEL D. BERNAL ${ }^{1-4}$, ENRIQUE T. ONA ${ }^{2}$, AILEEN RIEGO-JAVIER ${ }^{2}$, ROMULO DE VILLA $^{2}$, \\ GLORIA R. CRISTAL-LUNA ${ }^{2}$, JOSEPHINE B. LAGUATAN ${ }^{4}$, EUNICE R. BATAC ${ }^{4}$ and OSCAR Q. CANLAS ${ }^{4}$ \\ ${ }^{1}$ Regenerative Medicine Center, The Medical City; ${ }^{2}$ Center for Molecular and Cellular Therapeutics, \\ National Kidney and Transplant Institute, Quezon City, Philippines; ${ }^{3}$ Section of Hematology/Oncology, \\ Cedars-Sinai Medical Center, University of California, Los Angeles; ${ }^{4}$ GlobeTekPro, Woodland Hills, CA, USA
}

Received May 16, 2011; Accepted August 26, 2011

DOI: $10.3892 / \mathrm{ol} .2011 .424$

\begin{abstract}
Hematopoietic stem cells collected by leukapheresis of a patient with metastatic ovarian carcinoma (OVCA) were induced into dendritic cell (DC) differentiation and fused with liposomal constructs of autologous and allogeneic ovarian carcinoma antigens (DC-OVCA). The proliferation of autologous $\mathrm{T}$ cells induced by DCs was determined by $\left[{ }^{3} \mathrm{H}\right]$-thymidine uptake. Maximal T-cell proliferation was observed in co-cultures of DCs fused with liposomal OVCA constructs compared with intact autologous OVCA cells. The combination of autologous and allogeneic liposomal OVCA constructs induced greater T-cell proliferation than either alone. The cytotoxicity of DC-activated $\mathrm{T}$ cells against various target cells were analyzed by a ${ }^{51} \mathrm{Cr}$-release assay. The combination of autologous and allogeneic liposomal OVCA constructs showed the highest stimulation of T cell-mediated cytotoxicity against OVCA cells, but had minimal cytotoxicity against normal fibroblasts or leukemia cells. The liposomal preparations of DC-OVCA were injected monthly into a patient with metastatic ovarian carcinoma whose tumors progressed following multiple courses of chemotherapy. DCs analyzed from the patient post-immunization showed 2- to 3-fold greater OVCA cytotoxicity compared to pre-immunization DCs. Immunoblots using the patient's serum showed reactivity with a number of proteins from ovarian cancer extracts, but not in normal fibroblasts and breast cancer. Following the DC-OVCA treatment, the metastatic lesions progressively decreased in size to the point of being undetectable by serial CAT scans. Seven years following the initial diagnosis, the patient continues to be free of cancer. This report described the anticancer immune reactivity and anti-tumor response induced
\end{abstract}

Correspondence to: Dr Samuel Bernal, GlobeTekPro, 20000 Calvert Street, Woodland Hills, CA 91567, USA

E-mail: sberne@ucla.edu

Key words: cancer, immune reactivity, long-term survival, cancer treatment, metastatic ovarian cancer, ovarian cancer, dendritic cells, anticancer, cancer treatment, regenerative medicine by DCs sensitized with liposomal constructs of OVCA antigens. Immune cell therapy may therefore be a useful adjunct to surgery and chemotherapy for the treatment of ovarian cancer.

\section{Introduction}

Ovarian cancer (OVCA) remains the leading cause of mortality for patients with gynecologic malignancies, despite recent advances in post-surgical chemotherapy (1). Numerous patients with OVCA are diagnosed at an advanced stage of the disease, but may be treated with surgery followed by chemotherapy. Initially, OVCA may be chemotherapy-sensitive and the majority of patients achieve clinical remission following primary surgical debulking and adjuvant platinum-based therapy. However, the majority of patients who respond to such therapy ultimately succumb due to relapse from recurrent and drug-resistant disease. Following initial treatment, $70-90 \%$ of patients relapse. With additional second-line chemotherapy, some patients return to partial or complete remission. However, further relapse is likely to occur (2).

Novel therapies that reduce the risk of relapse are therefore urgently required. A variety of systemic and regional consolidation approaches with standard chemotherapy are being investigated. In this context, targeted immunotherapy may be a useful anti-OVCA strategy since such therapy may be effective against chemotherapy-resistant disease with minimal toxicity. This observation has also led to the recent development of various immunotherapeutic treatment strategies (3).

One of the requirements for this approach is the identification of antigens capable of mediating tumor rejection. Certain antigen targets for immunotherapy of ovarian cancer such as MUC1 $(4,5)$, Her-2/neu $(6,7)$ and CA125 $(8,9)$ are already known. However, other antigens remain to be identified. The majority of tumor antigens are non-mutated self-antigens to which the immune system is tolerant. It is well documented that tumor cells do not express co-stimulatory molecules and seldom express MHC class II molecules, which are both essential for the induction of cell immunity. As a result, tumor antigens may not be effectively presented to the immune system $(10,11)$, or worse, $\mathrm{T}$ cells responding to tumor antigens may be anergic (12-14). Therefore, new approaches are required to overcome these barriers that prevent the induction 
of effective anti-tumor immunity against antigens selective for, or over-expressed in, OVCA.

A strategy that has been developed is the sensitization of dendritic cells (DCs) with tumor cells or tumor antigens. DCs are bone marrow-derived leukocytes that are critical in the initiation of $\mathrm{T}$ cell-mediated immunity $(15,16)$. These cells derive their potency from the constitutive and inducible expression of essential co-stimulatory ligands on the cell surface, including CD80, CD86, CD56 and CD40 (17,18). DCs fused with patient-derived OVCA cells $(19,20)$ were found to induce cytotoxic $\mathrm{T}$ cells against autologous OVCA cells.

In the present study, we described the use of DCs sensitized with a liposomal construct of autologous and allogeneic tumor antigens (DC-OVCA) in a patient with metastatic OVCA who had residual metastatic lesions following chemotherapy and surgery.

\section{Materials and methods}

Consent for DC-OVCA injections. Informed consent was obtained from the patient prior to the collection of DCs and OVCA cells, and prior to injections. The consent form and immune cell procedures were reviewed and approved by the Ethics Committee and the Institutional Review Committee on Molecular and Cellular Therapeutics at the National Kidney and Transplant Institute, Philippines.

Generation of DCs from peripheral blood monocytes. Leukapheresis was performed for $4 \mathrm{~h}$ using a Cobe leukapheresis machine. The peripheral blood monocytes (PBMC) were separated through Ficoll density-gradient centrifugation, washed twice with RPMI-1640, and resuspended in RPMI-1640 with $1 \%$ autologous serum. Cells were cultured in a $\mathrm{CO}_{2}$ incubator overnight in sterile culture flasks. Following the initial culture, the non-adherent cells were decanted and the remaining adherent cells were cultured in the presence of $5 \%$ autologous serum/RPMI with $1000 \mathrm{U} / \mathrm{ml}$ GM-CSF and $500 \mathrm{U} / \mathrm{ml} \mathrm{IL}-4$ for 10 days. The cells were then trypsinized and resuspended in RPMI-1640, 40\% autologous serum and $10 \%$ DMSO. The cells were transferred to freezing vials, progressively cooled to lower temperatures, and then frozen in liquid nitrogen.

Isolation and culture of OVCA cells. OVCA cells were isolated from the resected specimen at the time of surgery. Tumor samples were transferred to the laboratory in a gentamycincontaining RPMI-1640. The tumor tissue $(1 \mathrm{mg} / \mathrm{ml})$ was minced finely with a sterile scalpel, transferred into a sterile petri dish containing RPMI-1640 with L-glutamine, gentamycin and $10 \%$ autologous plasma at a concentration of $5 \times 10^{6}$ tumor cells/ $\mathrm{ml}$, and cultured in a $5 \% \mathrm{CO}_{2}$ incubator at $37^{\circ} \mathrm{C}$. After three days, the adherent cells were plated in sterile flasks containing RPMI-1640 with L-glutamine, gentamycin and $10 \%$ autologous plasma, and cultured further in a $5 \% \mathrm{CO}_{2}$ incubator at $37^{\circ} \mathrm{C}$. After seven days, approximately half of the cultured cells were trypsinized and frozen in a medium containing 50\% autologous serum, 40\% RPMI-1640 and 10\% DMSO.

Characterization of DCs and OVCA cells. After 10 days in culture, a portion of DCs were trypsinized and stained by indirect immunofluorescence with a CD86 antibody (Pharmingen, San Diego, CA, USA), which is reactive with DCs. The autologous and allogeneic OVCA cells were tested by indirect immunofluorescence with antibodies against intracellular keratin and Her2/neu (Sigma, St. Louis, MO, USA), CA125 (Novocastra, Newcastle, UK) and MUC1 (Pharmingen) after seven days.

Fusion of DCs with liposomal construct of autologous and allogeneic OVCA antigens. The OVCA cells isolated above were cultured for seven days and irradiated at $50 \mathrm{~Gy}$. The autologous OVCA cells $\left(2 \times 10^{6}\right)$ were mixed with MUC-positive allogeneic OVCA cells $\left(1 \times 10^{6}\right)$ at a ratio of 2:1, respectively. This mixture was washed twice with serum-free RPMI-1640. The cell mixture was centrifuged at $2000 \mathrm{rpm}$ and resuspended in serum-free RPMI-1640. The cell pellet was sonicated for $5 \mathrm{~min}$ and resuspended in $0.50 \mathrm{ml}$ of a liposomal mixture of phosphatidylcholine and phosphoethanolamine containing 5\% polyethylene glycol (PEG) solution without $\mathrm{Ca}^{++}$and $\mathrm{Mg}^{++}$, and maintained at $37^{\circ} \mathrm{C}$ for $5 \mathrm{~min}$. The liposomal construct of OVCA antigens was mixed with $1 \times 10^{7}$ autologous DCs, centrifuged and resuspended in $1.5 \mathrm{ml}$ of RPMI-1640 with $10 \%$ autologous plasma. PEG-induced fusion was confirmed by microscopy. For preparation of the DC-OVCA injection, the DCs fused with liposomal OVCA were mixed with $500 \mathrm{U} / \mathrm{ml}$ GM-CSF placed in two sterile syringes containing $0.75 \mathrm{ml}$ of the mixture per syringe for intradermal injection.

In vitro tests of immune activity and T-cell proliferation. PBMC of the patient was isolated by Ficoll density-gradient centrifugation. PBMC was cultured in RPMI-1640 containing $1 \%$ autologous serum for $1 \mathrm{~h}$. The non-adherent cells were removed, and the $\mathrm{T}$ cells were purified by nylon wool separation. DCs (isolated from the patient prior to in vivo immunization) were treated with mitomycin $\mathrm{C}$ at $50 \mu \mathrm{g} / \mathrm{ml}$ for 45 min and washed 3 times with PBS. The DCs were resuspended in RPMI-1640 with $20 \%$ fetal calf serum. The T cells were co-cultured with DCs at a 10:1 ratio of T cells to DCs in medium containing $10 \%$ human serum for five days. T-cell proliferation was measured using standard $\left[{ }^{3} \mathrm{H}\right]$-thymidine incorporation. The cells were pulsed with $1 \mathrm{uCi}\left[{ }^{3} \mathrm{H}\right]$-thymidine (New England Nuclear, Boston, MA, USA) per well for $12 \mathrm{~h}$ and then collected on filters with a semi-automatic cell harvester. Tritium incorporation was quantified by liquid scintillation. Determinations were conducted in triplicate and expressed as the mean $\pm \mathrm{SD}$.

Cytolytic (CTL) activity directed against OVCA cells. PBMC $\left(10 \times 10^{6}\right)$ from the patient was cultured in RPMI-1640 containing $1 \%$ autologous serum for $1 \mathrm{~h}$. The $\mathrm{T}$ cells were purified by passage using nylon wool. DCs (isolated from the patient prior to in vivo immunization) sensitized with various antigen constructs were added at a 10:1 ratio of T cells to DCs in medium containing $10 \%$ human serum in the presence of $1000 \mathrm{U} / \mathrm{ml} \mathrm{GM-CSF}$ and $500 \mathrm{U} / \mathrm{ml} \mathrm{IL-4}$ for 10 days. The sensitized $\mathrm{T}$ cells were then co-cultured with ${ }^{51} \mathrm{Cr}$-labeled targets for $5 \mathrm{~h}$ at $37^{\circ} \mathrm{C}$. The cell targets included normal fibroblasts, autologous OVCA cells, allogeneic OVCA cells and the HL60 leukemia cell line. The T cells and various cell targets 
were resuspended in culture medium at a 10:1 ratio of $\mathrm{T}$ cells to target cells in 96-well, V-bottom plates. The plates were centrifuged at $1400 \mathrm{rpm}$ for $5 \mathrm{~min}$ to initiate cell contact and incubated for $5 \mathrm{~h}$ at $37^{\circ} \mathrm{C}$ with $5 \% \mathrm{CO}_{2}$. Following incubation, supernatant was collected and radioactivity was quantified in a gamma counter. The spontaneous release of ${ }^{51} \mathrm{Cr}$ was determined by incubation of targets in the absence of effectors, while maximum or total release of ${ }^{51} \mathrm{Cr}$ was determined by the incubation of targets in $0.1 \%$ Triton X-100. The percentage of specific release of ${ }^{51} \mathrm{Cr}$ was determined using the equation: percentage-specific release $=[($ experimental - spontaneous $) /$ (maximum - spontaneous)] x 100.

Immunoblotting of patient serum on OVCA antigens. Membrane extracts of primary foreskin fibroblasts, autologous OVCA cells isolated from the patients, the OVCAR cell line (ATCC) and the MCF7 breast carcinoma cell line (ATCC) were standardized to a protein concentration of $10 \mu \mathrm{g} / \mathrm{ml}$ and applied to SDS-PAGE samples, and were then separated on SDS-PAGE and transferred onto a Hybond-C nitrocellulose membrane (Amersham, UK). The membrane was blocked with $5 \%$ non-fat milk in TBST $(10 \mathrm{mM}$ Tris $\mathrm{HCl}, \mathrm{pH} 7.5$, $100 \mathrm{mM} \mathrm{NaCl}$ and $0.1 \%$ Tween-20) and incubated with the patient's serum overnight at $4^{\circ} \mathrm{C}$. After the membrane was exposed to goat anti-human secondary antibodies for $1 \mathrm{~h}$, the blots were detected using an enhanced chemiluminescence method (Pierce, Rockford, IL, USA).

\section{Results}

Patient data. DCs generated from a patient with metastatic OVCA were sensitized with different antigenic constructs in vitro and analyzed for immune reactivity. DCs isolated from the patient prior to and following immunization with DC-OVCA were analyzed for cytotoxicity against OVCA and other cell targets in vitro. The immune reactivity of the patient prior to and following DC-OVCA administration was analyzed by the immunoblot recognition of OVCA antigens. The clinical response of the patient was then followed by serial CAT scans.

The patient was a 35-year-old physician with a gynecological history of G2P2Ab0. The patient was generally asymptomatic with the exception of occasional pelvic pains that started in mid 2002. The patient took oral contraceptives for a few weeks in 2002, which decreased the frequency of her pelvic pains. In February 2004, she developed increasing cramping pain in the pelvic area associated with loose stools. An abdominal ultrasound performed on March 17, 2004, showed suspicious liver lesions. A CT scan on March 18, 2004, showed multiple pelvic masses, the largest measuring $5.6 \times 4.8 \mathrm{~cm}$. Multiple lesions were observed in the liver, the largest measuring $4.7 \mathrm{~cm}$ in the left lobe of the liver. In addition, there were numerous pulmonary masses involving the lungs. Lower GI endoscopy on March 27, 2004, was normal with the exception of hemorrhoids. Upper GI endoscopy on March 28, 2004, was normal and the stomach biopsy was negative. The mammogram and thyroid ultrasound were also normal. Serum tumor markers obtained on March 29, 2004, included a CA125 of $41 \mathrm{U} / \mathrm{ml}$ (normal, $<35 \mathrm{U} / \mathrm{ml}$ ), CA 19-9 of $46 \mathrm{U} / \mathrm{ml}$ (normal, <27 u/ml), CEA of $6.3 \mathrm{ng} / \mathrm{ml}$ (normal, $<5.5 \mathrm{ng} / \mathrm{ml}$ ), AFP was $4 \mathrm{IU} / \mathrm{ml}$ (normal, $<5.8 \mathrm{IU} / \mathrm{ml}$ ) and HCG was negative. On April 4, 2004, the patient underwent a CT-guided liver biopsy which revealed metastatic and poorly differentiated adenocarcinoma. Immunohistochemical stains were positive for CK7, but negative for CK20, TTF-1, S-100, ER, chromogranin, placental alkaline phosphatase, hepar-1 and inhibin. The diagnostic impression at this time was metastatic adenocarcinoma of unknown primary origin.

Treatments. In April 2004, the patient started chemotherapy consisting of carboplatin with AUC 7, paclitaxel $175 \mathrm{mg} / \mathrm{m}^{2}$, and etoposide $50 \mathrm{mg}$ alternating with $100 \mathrm{mg}$ days $1-10$, administered every 3-4 weeks. Following the sixth course of chemotherapy, the patient developed persistent thrombocytopenia. The etoposide dose was reduced in subsequent courses and eventually discontinued. After six courses of chemotherapy, CT scans on September 29, 2004, showed numerous residual masses, with the largest liver lesion measuring $3.6 \times 2.0 \mathrm{~cm}$ and the largest pelvic masses measuring $3.7 \times 3.0 \mathrm{~cm}$ in diameter. Chemotherapy was continued with carboplatin and paclitaxel for four additional courses. A CT scan on December 28, 2004, showed multiple liver lesions with the largest still measuring $3.6 \times 2.0 \mathrm{~cm}$, and bilateral multiple masses in the lungs with each measuring approximately $1 \mathrm{~cm}$ in diameter. However, certain lesions increased in size despite continued chemotherapy. A right adnexal mass increased in size to $5.7 \times 4.4 \mathrm{~cm}$, almost double in size compared to that of three months prior to the measurement.

In January of 2005, the patient complained of increasing right pelvic pain requiring narcotics. An MRI performed on January 20, 2005, showed a further enlargement of the largest adnexal mass, now measuring $7.2 \times 4.9 \times 4.2 \mathrm{~cm}$. Multiple metastatic tumors were also observed in the liver and lungs. Due to intractable pelvic pain, the patient underwent palliative surgery, with total hysterectomy with bilateral salpingo-oophorectomy on January 5, 2005. At the time of surgery, a large cystic mass was found in the right ovary. The histological diagnosis was endometrioid adenocarcinoma with a tubulo-papillary pattern arising from an ovarian cyst (Fig. 1). Numerous multinucleated giant cells were also found in the tissue specimen (Fig. 2). Part of the OVCA was minced, frozen, and used as intact cells or as cell lysate incorporated into a liposomal construct.

From the resected ovarian mass, viable OVCA cells were isolated and cultured for two weeks until processing for antigen. Aliquots of the cultured cells were allowed to adhere to sterile coverslips and were tested for antigen reactivity. Permeabilized cells were reactive with anti-cytokeratin in the cytoplasm. There was strong membrane staining with MUC1, weak staining for CA125 and negative staining for the Her2/neu antigen. Following 2 weeks in culture, the adherent cells were trypsinized and irradiated at $50 \mathrm{~Gy}$ prior to use as antigen either as intact cells or as a tumor lysate.

The patient underwent a 4-h leukapheresis without any complications. The leukapheresis sample, following Ficoll density-gradient centrifugation, yielded a total of $5 \times 10^{7}$ mononuclear cells. Following the initial culture, the non-adherent cells were decanted and the adherent population was incubated further in the presence of 5\% autologous serum and RPMI premixed with $1000 \mathrm{U} / \mathrm{ml}$ GM-CSF and $500 \mathrm{U} / \mathrm{ml} \mathrm{IL-4.}$ 


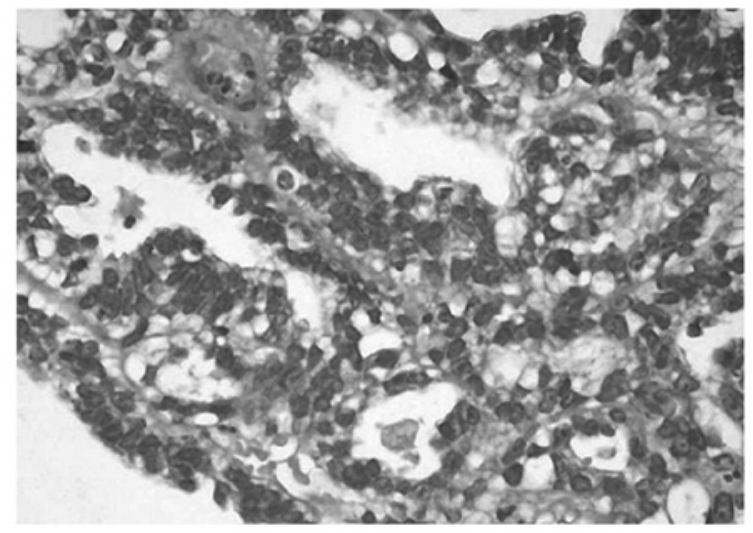

Figure 1. Endometrioid adenocarcinoma with tubulo-papillary pattern in an ovarian cyst. H\&E stain. Magnification, $x 40$.

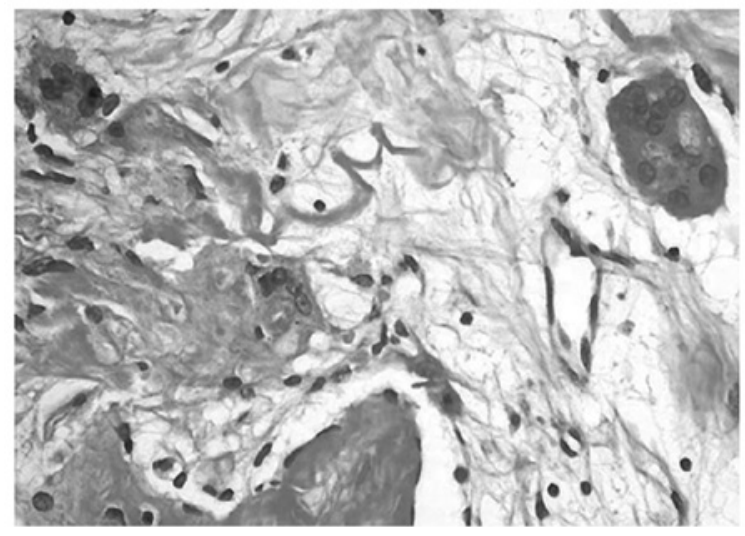

Figure 2. Multinucleated giant cells in an endometrioid adenocarcinoma with tubulo-papillary pattern. H\&E stain. Magnification, x100.

By five days, the cells flattened and became markedly adherent to the culture flask. By seven days, the majority of the cells exhibited long dendrite-like projections that came into contact with other cells. Approximately $80 \%$ of the cells were reactive with anti-CD86 antibody by indirect immunofluorescence microscopy. Following 10 days of culture, the cells were scraped gently and aliquoted into vials with RPMI containing 10\% DMSO. After the serial temperature dropped, the vials were stored in a liquid nitrogen tank. Two vials were thawed after five days in the frozen state and found to have $70 \%$ viability by trypan blue compared to $95 \%$ viability prior to freezing.

T-cell proliferation induced by the fusion of DC. In vitro tests of immune reactivity were performed on DCs developed from the mononuclear cells collected by leukapheresis. Various concentrations of control liposome, autologous OVCA cells, liposomal construct of autologous OVCA, and liposomal construct of allogeneic OVCA were fused with the DCs and tested for their ability to stimulate T-cell proliferation. As shown in Fig. 3, liposomes in the absence of antigens did not significantly induce T-cell proliferation. DCs fused with autologous whole OVCA cells showed increasing T-cell proliferation, up to $60 \mu \mathrm{g} / \mathrm{ml}$ of the construct, but reached a plateau. Higher concentrations of the antigen preparations did not induce greater T-cell proliferation. A liposomal construct

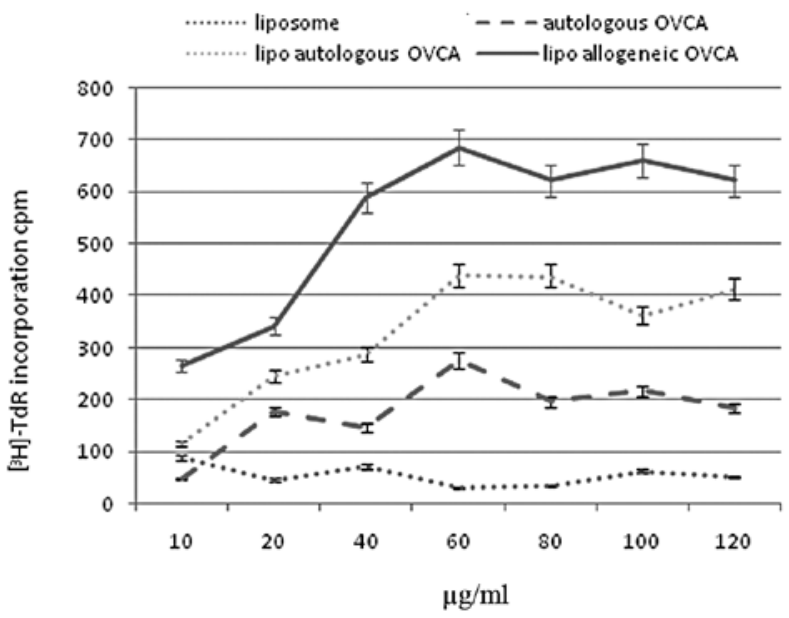

Figure 3. T-cell proliferation induced by the fusion of DC with different concentrations of control liposomes, autologous OVCA cells, liposomal construct of autologous OVCA and liposomal construct of allogeneic OVCA.

[ [ $\left.{ }^{3} \mathrm{H}\right] \cdot \mathrm{T} \mathrm{dR}$ incorporation $\mathrm{cpm}$

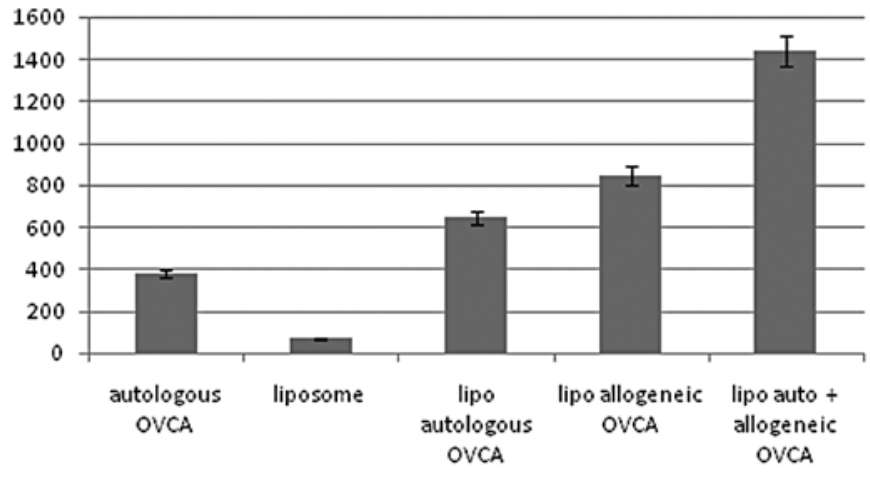

Figure 4. T-cell proliferation induced by fusion of DC with (A) autologous OVCA, (B) control liposome, (C) liposomal autologous OVCA, (D) liposomal allogeneic OVCA and (E) a combination of liposomal autologous and liposomal allogeneic OVCA.

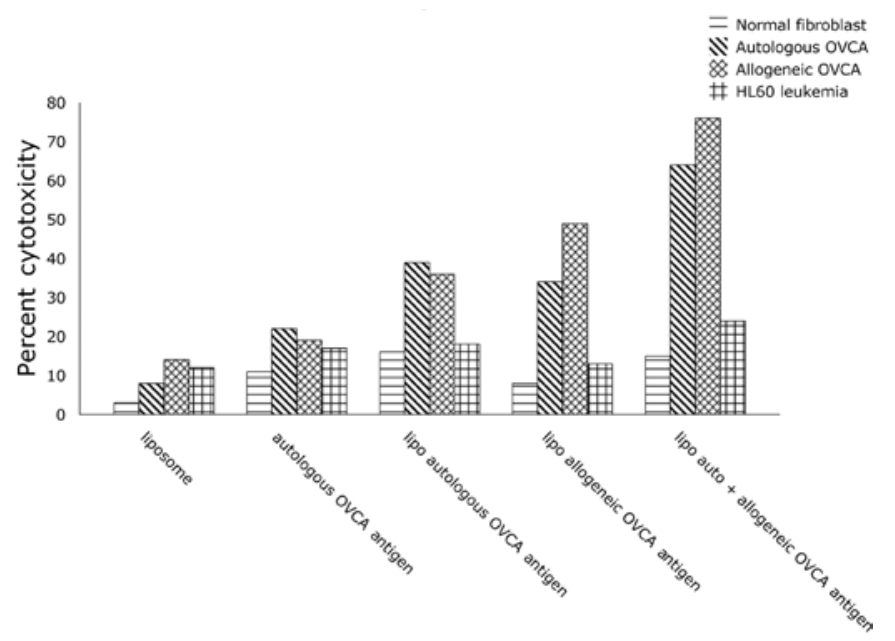

Antigen construct

Figure 5. In vitro cytotoxicity induced by fusion of DC with (A) liposome, (B) autologous OVCA, (C) liposomal autologous OVCA, (D) liposomal allogeneic OVCA, and (E) liposomal autologous and allogeneic OVCA. 
of autologous OVCA antigens showed a higher T-cell proliferation, almost 2-fold compared to intact autologous OVCA cells. A liposomal construct of allogeneic OVCA cells showed an even higher T-cell proliferation, almost 3-fold compared to the intact autologous OVCA cells.

Using the peak concentration $(60 \mu \mathrm{g} / \mathrm{ml})$ of each construct, T-cell proliferation induced after fusion with DCs was determined using the combination of liposomal autologous and liposomal allogeneic OVCA. The results, shown in Fig. 4, demonstrated that further stimulation of T-cell proliferation was induced by the liposomal construct combining autologous and allogeneic OVCA antigens with a higher activity compared with autologous OVCA, control liposomes, or single constructs of liposomal autologous OVCA, or liposomal allogeneic OVCA alone.

In vitro cytotoxicity induced by fusion of $D C$. The effect of fusion of DCs with different antigen preparations on the cytotoxicity of $\mathrm{T}$ cells in vitro was determined. As shown in Fig. 5, the greatest OVCA cell cytotoxicity was induced by fusion of DCs with a liposomal construct combining autologous and allogeneic OVCA antigens compared with autologous intact OVCA cells, or single liposomal constructs of autologous OVCA or allogeneic OVCA alone. Control liposomes without OVCA antigens had minimal effect on T-cell induction of cytotoxicity.

The immunogen administered to the patient consisted of DCs fused with a liposomal construct of autologous and allogeneic OVCA antigens. The patient received six monthly intradermal injections (January to June, 2005) of DC-OVCA, in each arm. With the exception of mild local skin reactions of swelling and slight redness that lasted for two days, the patient tolerated the injections well without fever, rash, muscle aches or joint pains. Following the six monthly injections, the patient's CT scans showed progressive reductions in the size and number of the metastatic lesions in the liver, lungs and pelvis. Since the patient still had residual masses, she received two additional chemotherapy courses with carboplatinum and taxol but was unable to continue due to severe thrombocytopenia, neutropenia, nausea and neuropathy. The patient's last chemotherapy cycle was administered in July 2005.

The patient was subsequently treated with DC-OVCA every month for an additional four treatments, without any chemotherapy. The patient showed continued improvement in her strength and peripheral nerve symptoms. Moroever, she gained weight and her overall function improved. A follow-up CT scan of the abdomen and pelvis in September 2005, showed a marked decrease in the number and size of metastatic lesions in the liver. The pelvis showed no masses. Lung CT showed a decreased size and number of lung nodules.

Patient outcome. By January 2006, a CT scan of the patient's lungs, liver and pelvis showed no metastatic lesions (Fig. 6-8). The patient was asymptomatic, maintaining normal activities with normal blood tests two years following the diagnosis of metastatic ovarian cancer. In 2006, the patient's only treatment consisted of one OVCA immune injection. On recent followup, the CT scans performed in March and November of 2006, and January 2007, showed no tumor masses. Serum tumor markers, including CA125, and liver functions tests were all normal. On further clinical follow-up and scans in 2010 and 2011, seven years following diagnosis, the patient remained tumor-free, asymptomatic and with a normal lifestyle.

After the patient completed DC-OVCA immunization, mononuclear cells were isolated to prepare DCs and were compared with pre-immunization DCs. As shown in Fig. 9, the post-immunization DCs showed 2- to 3-fold greater toxicity against autologous and allogeneic OVCA cells compared to pre-immunization DCs. The post-immunization and preimmunization DCs did not show any significant cytotoxicity against normal fibroblasts or HL60 leukemia cells.

The patient's serum collected in January 2006, following completion of the DC-OVCA treatment was examined by immunoblot analysis, which showed that the serum antibodies were reactive antigens of $60,65,110$ and $120 \mathrm{kDa}$ present in autologous OVCA (Fig. 10B) and allogeneic OVCA cells (Fig. 10D). The serum collected prior to DC-OVCA did not show reactivity with these antigens (data not shown). The serum antibodies upon completion of DC-OVCA treatment did not react with similar sized antigens in extracts from normal fibroblasts (Fig. 10A) and MCF 7 breast cancer cells (Fig. 10C).

\section{Discussion}

Present treatments of OVCA. Epithelial OVCA is the sixth most common cause of mortality due to cancer worldwide. The overall prognosis is poor as most patients present with advanced disease and efforts for early detection and screening have not been effective. The patient reported in this study is a young physician who only had a few months of mild pelvic discomfort, but at initial diagnosis already had large pelvic masses and numerous metastatic lesions in the lungs and the liver. The current 'standard treatment' for advanced ovarian cancer is a combination of a platinum compound and a taxane, usually in the form of carboplatin and paclitaxel (21). Although this platinum/taxane-based chemotherapy produces a high regression rate, the large majority of patients (70-90\%) relapse within 18 months, often with chemotherapy-resistant diseases. Overall survival is relatively poor with only $10-15 \%$ of advanced-disease patients surviving at 10 years. Therefore, new treatment approaches are required to improve response rates, to prevent relapse following chemotherapy and to prolong progression-free survival.

Rationale for immunotherapy in OVCA. Evidence exists that tumor antigens and anti-tumor immunity exist in patients with OVCA and affect the course of the cancer (22-25). A number of known tumor-associated antigens, including MUC-1, Her2/ neu, p53, CA125, MAGE antigens and testis cancer antigens, were found to be overexpressed in OVCA (26). Tumor antigen-specific $\mathrm{T}$ cells occur naturally in OVCA patients. Naturally occurring $\mathrm{T}$ cells that recognize tumor-associated antigens were found in 50\% of OVCA patients (27). The presence of intratumoral $\mathrm{T}$ cells correlates with improved clinical outcome in advanced OVCA (24). These tumor-infiltrating $\mathrm{T}$ cells are capable of identifying autologous ovarian tumor or peptides derived from OVCA tumor antigens $(22,23)$. The tumor antigen-specific $\mathrm{T}$ cells may be expanded from OVCA 
A

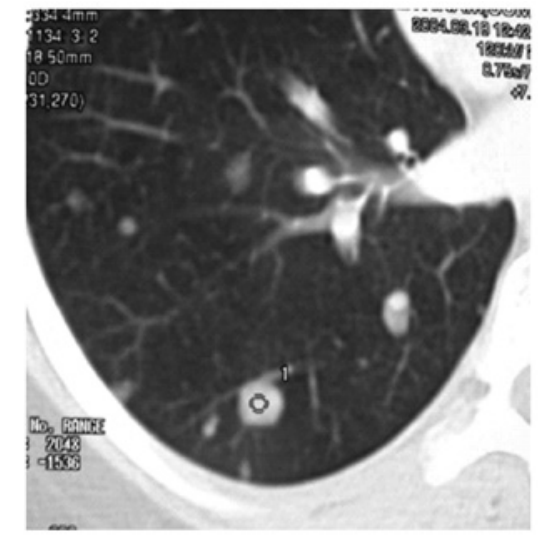

B

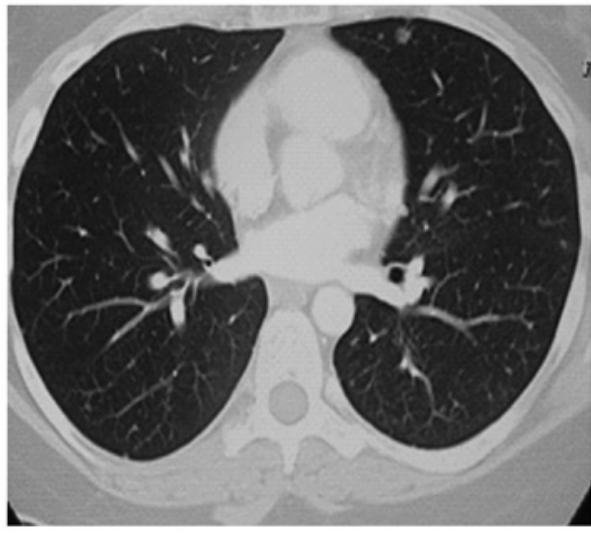

Figure 6. (A) March 2004. (B) Jan 2006.

A

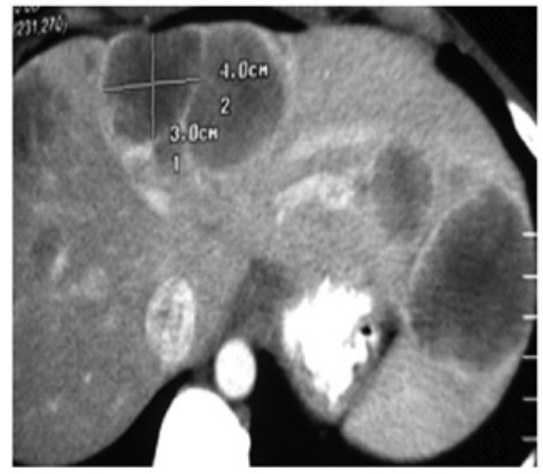

B

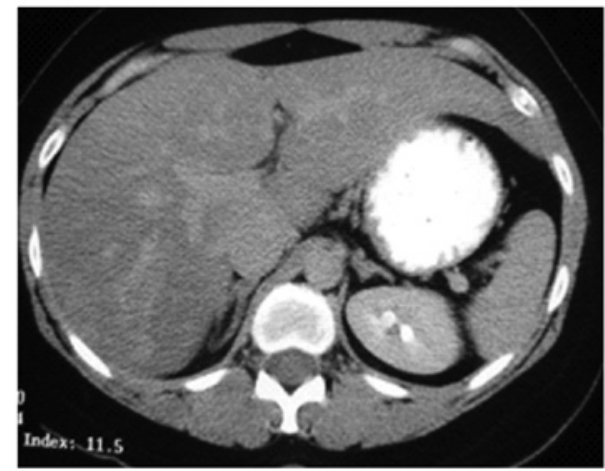

Figure 7. (A) March 2004. (B) Jan 2006.

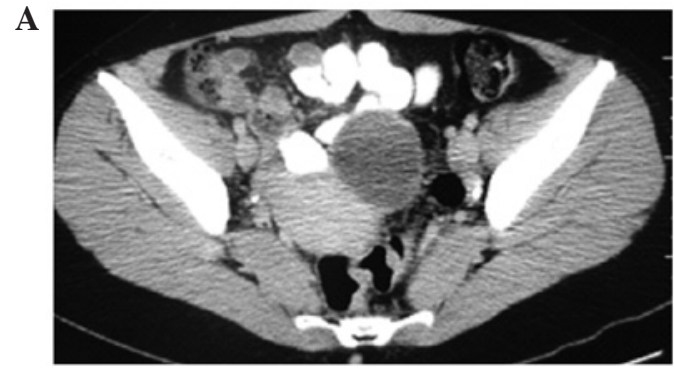

B

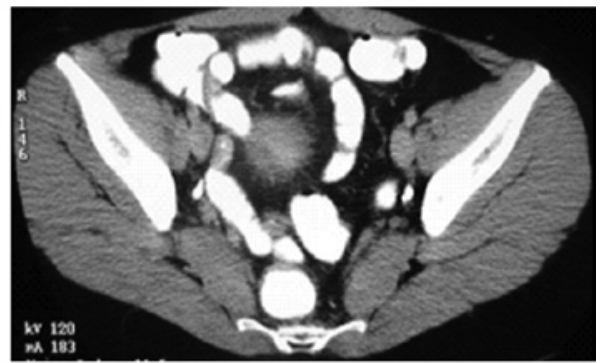

Figure 8. (A) March 2004. (B) Jan 2006.

patients in vitro (27) and may recognize similar epitopes in HER2/neu antigens of breast cancer (7). The adoptive transfer of these tumor-infiltrating lymphocytes has been found to prolong disease-free survival in patients with advanced OVCA (25).

The presence of tumor-infiltrating $\mathrm{T}$ cells was found to have a significant impact on the outcome of OVCA following chemotherapy (24). The five-year survival rate of patients whose tumor contained tumor-infiltrating $\mathrm{T}$ cells was $38 \%$, whereas the survival rate of patients without tumor-infiltrating $\mathrm{T}$ cells was only $4.5 \%$. This survival difference was highly statistically significant, with $\mathrm{p}<0.001$. The presence of T-cell infiltration was found by multivariate analysis to be a statistically independent prognostic variable that correlated with progression-free and overall survival, and was independent of the clinical response to chemotherapy.
Thus, there is strong justification, for exploring immunotherapy in the treatment of advanced OVCA using tumor antigen-specific cytotoxic immune cells for killing cancer cells by a mechanism distinct from chemotherapy $(28,29)$. Immunotherapy has the potential to enhance the body's own ability to attack the cancer with greater specificity and less toxicity. However, the appearance and growth of the tumor itself indicates a failure of the immune surveillance of the body at the control mechanisms for immune induction and action, and possibly indicates defects at the level of antigen presentation, the action of co-stimulatory molecules and immune tolerance, and $\mathrm{T}$ cell unresponsiveness to tumor-associated antigens.

The use of DCs is one way to enhance the tumor antigen presentation. In OVCA, the approaches for antigen presentation included DC-OVCA fusion and antigen pulsing $(30,31)$. DCs fused with OVCA cells have the potential of delivering 


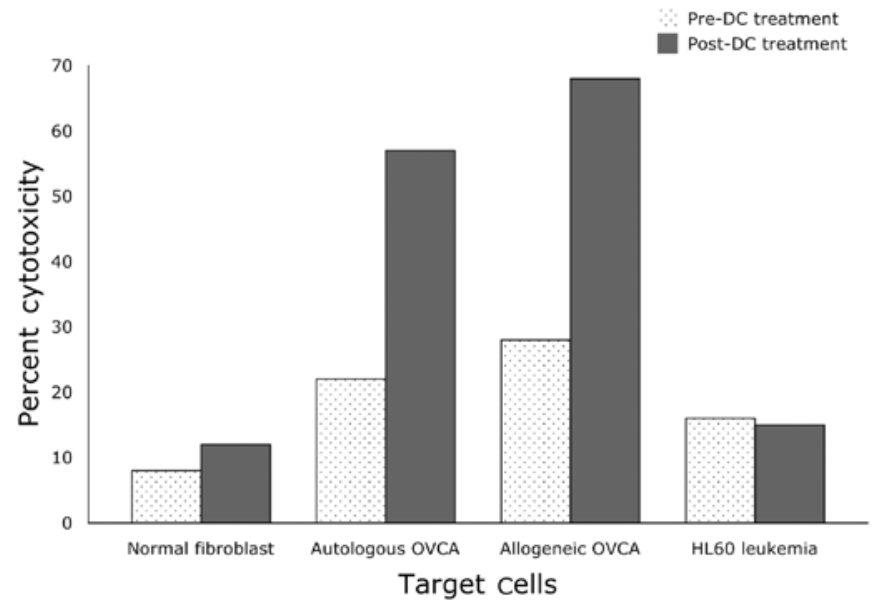

Figure 9. Target cell cytotoxicity of $\mathrm{T}$ cells isolated from the patient pre- and post-DC treatment.

the mature immune cells with the appropriate co-stimulatory and MHC molecules, and with the whole complement of tumor antigens to facilitate endogenous and exogenous pathways of antigen presentation, leading to the induction of CD4 and CD8 T cells. DCs/OVCA cells were found to stimulate the interferon expression of CD4 and CD8 T cells, and to induce CTL activity against autologous OVCA cells [A]. DC/ OVCA fusion cells stimulated $\mathrm{T}$ cells and reversed the unresponsiveness of $\mathrm{T}$ cells to autologous OVCA cells. The DCs of the patient are capable of being fused with autologous or allogeneic tumor cells, or both these types of cells.

In the current patient, viable autologous OVCA cells were collected at the time of surgery. The injections administered consisted of a liposomal construct including autologous and allogeneic OVCA cells. A significant advantage of using autologous tumor cells in the DC-OVCA fusion is that these cells contain the relevant OVCA antigens required to control the patient's tumor. Disadvantages include the need for arranging the collection of viable tumor at the time of surgery and the difficulties in culturing tumor cells. The culture of tumor cells requires advance planning, but since debulking surgery is often part of the treatment of OVCA, the opportunity for collecting and growing OVCA cells should not be missed.

When this patient underwent bilateral salpingooophorectomy and hysterectomy, she was found to have a large cystic mass in the right ovary with a histological diagnosis of endometrioid adenocarcinoma with a tubulo-papillary pattern arising from an ovarian cyst. The tumor cells were reactive with anti-cytokeratin in the cytoplasm, and had strong membrane staining for MUC1, weak staining for CA125 and negative staining for Her2/neu antigens. Although, histologically, the patient was found to have carcinoma arising from the ovary, there were no ovarian markers in the tissue and serum, such as CA125, that were useful in the identification of this patient's tumor. For this patient, tumor cells were kept viable at the time of surgery and were the source of autologous tumor cells used for DC sensitization. However, numerous patients with progressive ovarian cancer do not have viable tumor cells saved at the time of biopsy or surgery. Moreover, using only the patient's tumor cells may not effectively present antigens

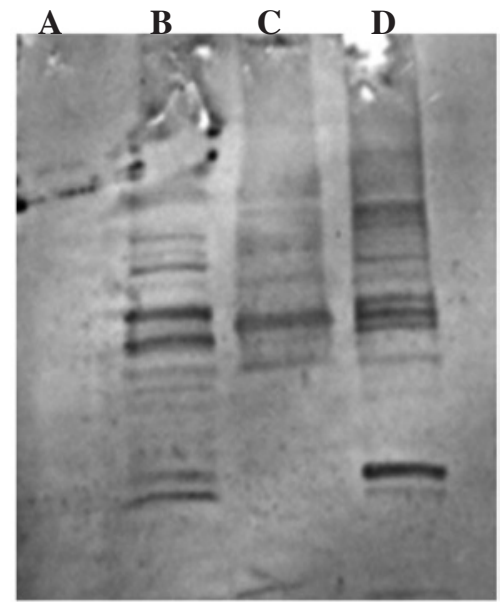

Figure 10. Immunoblotting using patient serum reactive with extracts of tumor cells. (A) Normal fibroblasts. (B) Autologous OVCA. (C) MCF7, breast cancer. (D) Allogeneic OVCA.

to the immune system $(10,11)$, and the patient's $\mathrm{T}$ cells may be anergic to the autologous tumor antigens (12-14). Therefore, for this patient, we also used allogeneic OCVA cells to supplement the autologous tumor cells.

Optimal immune reactivity in vitro and in vivo induced by the liposomal construct of autologous and allogeneic tumor antigens. To determine the best immunogen for DC sensitization, in vitro tests of immune activity were performed on the DCs and T cells collected at the time of initial leukapheresis. The peak concentrations of each preparation of intact OVCA cells, the liposomal construct of autologous OVCA antigens, and the liposomal construct of allogeneic OVCA antigens on T-cell proliferation were determined, showing higher immune activity using liposomal constructs compared to intact tumor cells. The T-cell proliferative activity using higher concentrations of each construct reached a plateau. However, the plateau was 2-fold higher with the liposomal construct of autologous OVCA antigens and approximately 3 -fold higher using the liposomal construct of allogeneic OVCA antigens compared to intact autologous OVCA cells. This observation emphasizes that simply fusing intact autologous tumor cells with DCs may not be sufficient to induce optimal immune reactivity against the tumor.

The highest T-cell proliferation was observed using a liposomal construct combining autologous and allogeneic OVCA cells. The highest OVCA target cell cytotoxicity was also observed with the liposomal construct with combined autologous and allogeneic OVCA antigens compared with intact autologous OVCA cells or liposomal constructs of autologous or allogeneic antigens alone. The cytotoxicity induced by the liposomal construct with combined autologous and allogeneic OVCA antigens induced much greater cytotoxicity on OVCA cells compared with normal fibroblasts and HL60 leukemic cells.

Based on the in vitro studies, the patient was injected with DCs fused with a liposomal construct with autologous and allogeneic OVCA antigens. The OVCA cells, both autologous and allogeneic, were irradiated at 50 Gy prior to being used in antigen extraction and incorporated into liposomes. Following 
completion of the DC-OVCA injections, DCs isolated from the patient post-immunization were compared to pre-immunization DCs. DCs prepared from mononuclear cells collected post-immunization showed 2- to 3-fold greater cytotoxicity against autologous and allogeneic OVCA cells compared to DCs pre-immunization. Serum antibodies collected postimmunization also showed reactivity with specific antigens present in OVCA cells, but were not present in normal fibroblasts and MCF-7 breast cancer cells. Thus, the immunization of this patient with a liposomal construct combining autologous and allogeneic OVCA cells induced specific immune reactivity against OVCA antigens in vivo.

Treatment with chemotherapy combined with DC-OVCA immunotherapy. The current patient was initially treated with chemotherapy prior to the DC-OVCA injections. At initial presentation, this patient was considered to have carcinoma of unknown primary origin. The initial CT scan showed numerous pulmonary masses involving the lungs, multiple pelvic masses and a number of large lesions in the liver. Lower GI and upper GI endoscopy were normal and the gastric biopsy was negative. The serum tumor markers at initial presentation, including CA125, CA 19-9 and CEA, were mildly elevated, whereas AFP and HCG were normal. A CT-guided liver biopsy revealed as a metastatic and poorly differentiated adenocarcinoma with no clear indication of primary origin. Immunohistochemical stains of the liver biopsy specimen were positive for CK7, but negative for CK20, TTF-1, S-100, ER, chromogranin, placental alkaline phosphatase, hepar-1 and inhibin. Thus, there were no germ cell markers present that would have predicted responsiveness to chemotherapy. The tumor was of epithelial origin but the formal interpretation of the initial biopsy was metastatic adenocarcinoma of unknown primary origin.

The patient's initial treatment regimen, based upon a diagnosis of carcinoma of unknown primary origin, consisting of carboplatinum, taxol and etoposide, caused considerable side effects, including abdominal discomfort, neuropathy, severe thrombocytopenia and neutropenia, requiring multiple dose adjustments, cytokine support and, eventually, discontinuation of the chemotherapy. Although the patient had a partial response to chemotherapy, she had multiple residual masses and progressive enlargement of pelvic masses. This mixed response to treatment was indicative of the heterogeneity of the tumor. Thus, chemotherapy by itself was not sufficient in controlling the tumor. The patient eventually underwent surgery to resect an enlarging pelvic mass, which caused increasing pelvic pain requiring narcotic administration.

The conventional view of chemotherapy is an immunosuppressive treatment that has the potential to reduce myelocytes and lymphocytes (32). However, patients with advanced OVCA were found to have enhanced T-cell function with increased $\mathrm{CD} 8+\mathrm{T}$-cell responses to a pool of $\mathrm{MHC}$ class I-restricted recall antigens in that following administration of carboplatinum-paclitaxel combination chemotherapy, patients with advanced OVCA were found to have enhanced T-cell function (31). Synergies between chemotherapy and immunotherapy have also been found in experimental tumor systems (33). Chemotherapy may act to decrease tumor load and tumor-induced immunosuppression, supporting the idea that the ideal time to induce anti-tumor immunity is following chemotherapy/radiation or debulking surgery.

In other types of gynecologic cancer, immune enhancement was found to be significant in the effects of chemotherapy. Patients who received platinum-based adjuvant chemotherapy and chemo-radiation for cervical cancer were also found to have an increased proportion of CD4+ and CD8+ T cells and natural killer cells in draining pelvic lymph nodes biopsied following surgery (34). The enhancement of immune cell activity was not limited to carboplatinum and paclitaxel. In an animal model, gemcitabine was found to increase antigen cross-presentation, T-cell expansion and infiltration of tumors, resulting in the eradication of the tumor. The effect of chemotherapy with gemcitabine did not appear to be due solely to decreased tumor bulk, as surgical resection of the tumor by itself did not lead to an enhanced immunotherapeutic effect (33).

By itself, immunotherapy alone is unlikely to be effective in cancer with bulky masses, such as ovarian cancer, even following surgery. Since chemotherapy enhances rather than impedes immune cell function, the best effect of immuno-therapy may be following chemotherapy. Moreover, immunotherapy may act better in minimal residual disease and may then be integrated in adjuvant treatment programs following surgery and/or radiation.

In conclusion, a combination of DC-OVCA treatment following chemotherapy was successful in treating a patient with metastatic OVCA who presented with large pelvic masses and large metastatic lesions in the liver and lungs. The patient had a partial response following chemotherapy but had persistent large masses in the pelvis and residual lesions in the liver and lungs. Following DC treatment, the patient's metastatic lesions markedly decreased in size. Seven years following the initial diagnosis, the patient has shown no residual disease. Thus, further investigations should be conducted on the use of immunotherapy in combination with chemotherapy in the treatment of OVCA.

\section{References}

1. Jemal A, Murray T, Samuels A, Ghafoor A, Ward E and Thun MJ: Cancer statistics. CA Cancer J Clin 53: 5-26, 2003.

2. McGuire WP, Hoskins WJ, Brady MF, Kucera PR, Partridge EE, Look KY, Clarke-Pearson DL and Davidson M: Cyclophosphamide and cisplatin compared with paclitaxel and cisplatin in patients with stage III and stage IV ovarian cancer. N Engl J Med 334: 1-6, 1996.

3. Kirby TO, Huh $\mathrm{W}$ and Alvarez R: Immunotherapy of ovarian cancer. Exp Opin Biol Ther 2: 409-417, 2002.

4. Ichige $\mathrm{K}$, Perey L, Vogel CA, Buchegger $\mathrm{F}$ and Kufe D: Expression of the DF3-P epitope in human ovarian carcinomas. Clin Cancer Res 1: 565-571, 1995.

5. Lu KH, Patterson AP, Wang L, et al: Selection of potential markers for epithelial ovarian cancer with gene expression arrays and recursive descent partition analysis. Clin Cancer Res 10: 3291-3300, 2004.

6. McKenzie SJ, DeSombre KA, Bast BS, et al: Serum levels of HER-2 neu (C-erbB-2) correlate with overexpression of p185neu in human ovarian cancer. Cancer 71: 3942-3946, 1993.

7. Peoples GE, Goedegebuure PS, Smith R, Linehan DC, Yoshino I and Eberlein TJ: Breast and ovarian cancer-specific cytotoxic T lymphocytes recognize the same HER2/neu-derived peptide. Proc Nat Acad Sci USA 92: 432-436, 1995.

8. Wagner U, Schlebusch H, Kohler S, Schmolling J, Grunn U and Krebs D: Immunological responses to the tumor-associated antigen CA125 in patients with advanced ovarian cancer induced by the murine monoclonal anti-idiotype vaccine ACA125. Hybridoma 16: 33-40, 1997. 
9. Jacobs I and Bast RC Jr: The CA 125 tumour-associated antigen: a review of the literature. Hum Reprod 4: 1-12, 1989.

10. Gusdon JP Jr, Homesley HD, Jobson VW and Muss HB: Treatment of advanced ovarian malignancy with chemoimmunotherapy using autologous tumor and Corynebacterium parvum. Obstet Gynecol 62: 728-735, 1983.

11. Dillman RO, Nayak SK, Brown JV, Mahdavi K and Beutel LD: The feasibility of using short-term cultures of ovarian cancer cells for use as autologous tumor cell vaccines as adjuvant treatment of advanced ovarian cancer. Cancer Biother Radiopharm 14: 443-449, 1999.

12. Bachmann MF, Speiser DE, Mak TW and Ohashi PS: Absence of costimulation and not the intensity of TCR signaling is critical for the induction of $\mathrm{T}$ cell unresponsiveness in vivo. Eur J Immunol 29: 2156-2166, 1999.

13. Chen L, Ashe S, Brady WA, et al: Costimulation of antitumor immunity by the B7 counterreceptor for the T lymphocyte molecules CD28 and CTLA-4. Cell 71: 1093-1102, 1992.

14. Zheng P, Sarma S, Guo Y and Liu Y: Two mechanisms for tumor evasion of preexisting cytotoxic T-cell responses: lessons from recurrent tumors. Cancer Res 59: 3461-3467, 1999.

15. Steinman RM: The dendritic cell system and its role in immunogenicity. Annu Rev Immunol 9: 271-296, 1991.

16. Steinman RM: Dendritic cells and the control of immunity: enhancing the efficiency of antigen presentation. Mt Sinai J Med 68: 106-166, 2001.

17. Young JW, Koulova L, Soergel SA, Clark EA, Steinman RM and Dupont B: The B7/BB1 antigen provides one of several costimulatory signals for the activation of CD4+ T lymphocytes by human blood dendritic cells in vitro. J Clin Invest 90 229-237, 1992.

18. Inaba $\mathrm{K}$, Witmer-Pack $\mathrm{M}$, Inaba $\mathrm{M}$, et al: The tissue distribution of the B7-2 costimulator in mice: abundant expression on dendritic cells in situ and during maturation in vitro. J Exp Med 180: 1849-1860, 1994

19. Gong J, Nikrui N, Chen D, et al: Fusions of human ovarian carcinoma cells with autologous or allogeneic dendritic cells induce antitumor immunity. J Immunol 165: 1705-1711, 2000.

20. Koido S, Ohana M, Liu C, et al: Dendritic cells fused with human cancer cells: morphology, antigen expression and $\mathrm{T}$ cell stimulation. Clin Immunol 113: 261-269, 2004.

21. Kaye S: Chemotherapy for ovarian cancer: yesterday, today and tomorrow. Br J Cancer 89: S1-S2, 2003.

22. Dadmarz RD, Ordoubadi A, Mixon A, et al: Tumor-infiltrating lymphocytes from human ovarian cancer patients recognize autologous tumor in an MHC Class II-restricted fashion. Cancer J Sci Am 2: 263, 1996.
23. Hayashi K, Yonamine $\mathrm{K}$, Masuko-Hongo $\mathrm{K}$, et al: Clonal expansion of $\mathrm{T}$ cells that are specific for autologous ovarian tumor among tumorinfiltrating $\mathrm{T}$ cells in humans. Gynecol Oncol 74: 86-92, 1999.

24. Zhang L, Conejo-Garcia JR, Katsaros D, et al: Intratumoral $\mathrm{T}$ cells, recurrence, and survival in epithelial ovarian cancer. N Engl J Med 348: 203-213, 2003.

25. Fujita K, Ikarashi H, Takakuwa K, et al: Prolonged disease-free period in patients with advanced epithelial ovarian cancer after adoptive transfer of tumor-infiltrating lymphocytes. Clin Cancer Res 1: 501-507, 1995.

26. Nijman $\mathrm{H}$, van Diest $\mathrm{P}$, Poort-Keesom R, et al: $\mathrm{T}$ cell infiltration and MHC I and II expression in the presence of tumor antigens: an immunohistochemical study in patients with serous epithelial ovarian cancer. Eur J Obstet Gynecol Reprod Biol 94: 114-120, 2001.

27. Freedman R and Platsoucas C: Immunotherapy for peritoneal ovarian carcinoma metastasis using ex vivo expanded tumor infiltrating lymphocytes. Cancer Treat Res 82: 115-146, 1996.

28. Schlienger K, Chu C, Woo E, et al: TRANCE- and CD40 ligand matured dendritic cells reveal MHC class I-restricted T cells specific for autologous tumor in late-stage ovarian cancer patients. Clin Cancer Res 9: 1517-1527, 2003.

29. Pardoll D: Spinning molecular immunology into successful immunotherapy. Nat Rev Immunol 2: 227-238, 2002.

30. Koidoa S, Nikruib N, Ohanac M, Xiac J, Tanaka Y, Liuc C, Durfeec J, Lernerc A and Gong J: Assessment of fusion cells from patient-derived ovarian carcinoma cells and dendritic cells as a vaccine for clinical use. Gynecologic Oncology 99: 462-471, 2005.

31. Adams M, Navabi H, Croston D, Coleman S, Tabi Z, Clayton A, Jasani B and Mason MD: The rationale for combined chemo/ immunotherapy using a Toll-like receptor 3 (TLR3) agonist and tumour-derived exosomes in advanced ovarian cancer. Vaccine 23: 2374-2378, 2005.

32. Rosenberg S, Yang J and Restifo N: Cancer immunotherapy: moving beyond current vaccines. Nat Med 10: 909-915, 2004.

33. Nowak A, Robinson B and Lake R: Synergy between chemotherapy and immunotherapy in the treatment of established murine solid tumors. Cancer Res 63: 4490-4496, 2003.

34. Fattorossi A, Battaglia A, Ferrandina G, et al: Neoadjuvant therapy changes the lymphocyte composition of tumor-draining lymph nodes in cervical carcinoma. Cancer 100: 1418-1428, 2004. 